\title{
Clinical Laboratory
}

National Cancer Institute

\section{Source}

National Cancer Institute. Clinical Laboratory. NCI Thesaurus. Code C142432.

A laboratory that analyzes patient or subject samples collected as part of healthcare treatment or clinical research. 